INPLASY

PROTOCOL

To cite: Lin et al. Empathy and theory of mind in multiple sclerosis: a protocol for systematic review and metaanalysis. Inplasy protocol 202070029. doi:

10.37766/inplasy2020.7.0029

Received: 10 July 2020

Published: 10 July 2020

Corresponding author: ZhongQuan Yi

yizhongquan@163.com

Author Affiliation:

Affiliated Yancheng Hospital, School of Medicine

Support: Jiangsu commission of Health

Review Stage at time of this submission: Piloting of the study selection process.

Conflicts of interest:

The authors have no conflicts of interest to disclose.

\section{Empathy and theory of mind in multiple sclerosis: a protocol for systematic review and meta-analysis}

Lin, XG1; Zhang, XL2; Liu, QQ3; Zhao, PW4; Zhong, JG5; Pan, PL6; Wang, GD7; Yi, ZQ ${ }^{8}$.

Review question / Objective: The aim of this study is to conduct a systematic review and meta-analysis to characterize the performance of empathy and ToM in MS.

Condition being studied: Multiple sclerosis (MS) is an immune-mediated demyelinating disease of the central nervous system (CNS). Recently, numerous studies have shown that MS disrupts a number of social cognitive abilities, including empathy, theory of mind (ToM), and facial emotion recognition. In contrast to well-documented deficits in the core social cognitive domains of ToM and facial emotion recognition, it is not clear the broad and specific subcomponents of empathy processing affected. In addition, the specific subcomponents of ToM affected in MS are also unclear.

INPLASY registration number: This protocol was registered with the International Platform of Registered Systematic Review and Meta-Analysis Protocols (INPLASY) on 10 July 2020 and was last updated on 10 July 2020 (registration number INPLASY202070029).

\section{INTRODUCTION}

Review question / Objective: The aim of this study is to conduct a systematic review and meta-analysis to characterize the performance of empathy and ToM in MS.

Condition being studied: Multiple sclerosis (MS) is an immune-mediated demyelinating disease of the central nervous system 
(CNS). Recently, numerous studies have shown that MS disrupts a number of social cognitive abilities, including empathy, theory of mind (ToM), and facial emotion recognition. In contrast to welldocumented deficits in the core social cognitive domains of ToM and facial emotion recognition, it is not clear the broad and specific subcomponents of empathy processing affected. In addition, the specific subcomponents of ToM affected in MS are also unclear.

\section{METHODS}

Participant or population: Patients diagnosed with MS will be included in the study. Patients with other serious complications, a history of brain surgery, or other serious neurodegenerative diseases will be excluded from this study.

Intervention: We will mainly study the performance of empathy and ToM between MS patients and healthy controls.

Comparator: Healthy controls.

Study designs to be included: Case-control studies.

Eligibility criteria: Studies were considered eligible for inclusion if (1) the study compared MS participants to a matched healthy controls group, (2) the study had to assess empathy performance or ToM performance using standard measures, (3) sufficient data to calculate effect sizes and standard errors of the empathy or ToM were reported, (4) the study was published in a peer-reviewed journal in English. Studies were considered eligible for exclusion if (1) the study with the patient samples was overlapped with another one with a larger sample size, (2) the study lacked an HC group, (3) the study with a sample size under 10 will be excluded to ensure the reliability of the outcome, (4) the publication was not an original type, such as research protocols, letters, conference abstracts, reviews, and editorials.

Information sources: Two independent authors will search the following electronic databases: PubMed, Web of Science and Embase. The search is from inception to July 1st, 2020 with no restriction of publication dates. In addition, other resources will be searched manually, such as the references of all included studies.

Main outcome(s): Main results: the measures of empathy and ToM and the data used for calculating the effect sizes and standard errors of the empathy and ToM measures.

Additional outcome(s): Additional outcomes will include the other questionnaire of clinical symptoms of MS.

Quality assessment / Risk of bias analysis: We will use the Newcastle-Ottawa Quality Assessment Scale (NOS) to assess the quality of all included studies.

Strategy of data synthesis: Stata $\mathbf{1 5 . 0}$ software will be used for data analysis and quantitative data synthesis. The mean effect size (Hedge's g) and 95\% confidence intervals $(\mathrm{Cl})$ will be used to evaluate the performance of ToM and facial emotion recognition. For studies reporting more than one ToM task, pooled effect size and standard error value were calculated. Effect sizes $<0.5$ were considered small, between 0.5 and 0.8 moderate, and $>0.8$ large. When appropriate, data will be pooled across studies for meta-analysis using fixed or random effect models.

Subgroup analysis: If the heterogeneity of the results is high and the data are sufficient, we will perform a subgroup analysis on the data in order to find the cause of the large heterogeneity. Subgroup analysis will be performed according to clinical subtypes (such as clinically isolated syndrome, relapsing-remitting MS, progressive primary MS, and secondary progressive MS).

Sensibility analysis: If publication bias was found, we will apply the trim-and-fill method to provide effect sizes adjusted for publication bias.

Language: English. 


\section{Country(ies) involved: China.}

Keywords: multiple sclerosis, empathy, theory of mind, systematic review, metaanalysis, protocol.

Contributions of each author:

Author 1 - XiaoGuang Lin - The author drafted the manuscript.

Author 2 - XueLing Zhang - The author provided statistical expertise.

Author 3 - QinQin Liu - The author contributed to the development of the selection criteria, the risk of bias assessment strategy, and data extraction.

Author 4 - PanWen Zhao - The author contributed to the development of the selection criteria, the risk of bias assessment strategy, data extraction, and drafted the manuscript.

Author 5 - JianGuo Zhong - The author provided methodological support and software analysis guidance.

Author 6 - PingLei Pan - The author provided methodological support and software analysis guidance.

Author 7 - GenDi Wang - The author contributed to conception, design, data analysis, and writing the manuscript.

Author 8 - ZhongQuan Yi - The author contributed to conception, design, data analysis, and writing the manuscript. 